\title{
BMJ Open Associations of pregnancy-associated plasma protein-A level with essential hypertension and hypertensive disorders in pregnancy in Chinese population: a meta-analysis of 20 research studies involving 3332 individuals
}

\author{
Gaojun Cai, ${ }^{1}$ Bifeng Zhang, ${ }^{2}$ Weijin Weng, ${ }^{1}$ Liping Yang, ${ }^{1}$ Ganwei Shi, \\ Sheliang Xue, ${ }^{1}$ Xingli $\mathrm{Fu}^{3}$
}

To cite: Cai G, Zhang B, Weng W, et al. Associations of pregnancy-associated plasma protein-A level with essential hypertension and hypertensive disorders in pregnancy in Chinese population: a meta-analysis of 20 research studies involving 3332 individuals. BMJ Open 2015;5:e008210. doi:10.1136/bmjopen-2015008210

- Prepublication history for this paper is available online To view these files please visit the journal online (http://dx.doi.org/10.1136/ bmjopen-2015-008210).

GC, BZ and XF contributed equally.

Received 17 March 2015 Revised 5 August 2015 Accepted 20 August 2015

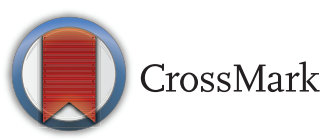

For numbered affiliations see end of article.

Correspondence to

Dr Gaojun Cai;

cgj982@126.com

\section{ABSTRACT}

Objective: To explore the associations between serum pregnancy-associated plasma protein-A (PAPP-A) level, and essential hypertension (EH) and hypertensive disorders in pregnancy (HDP) in Chinese population. Methods: Pertinent studies were independently searched in PubMed, Embase, Cochrane Library, Chinese Biomedical Database (CBM), Wanfang databases and China National Knowledge Infrastructure (CNKI). The standardised mean difference (SMD) with $95 \%$ Cls was used to estimate the size of the effect. The subgroup analyses and meta-regression analysis were performed to identify the sources of heterogeneity among studies. Sensitivity analysis was conducted to assess the stability of the results. The publication bias between studies was examined by using Begg's funnel plots and Egger's test.

Results: A total of 20 studies involving 1493 patients and 1839 controls were included in the current metaanalysis. The PAPP-A level was significantly higher in EH patients than in controls $(S M D=1.960,95 \% \mathrm{Cl}$ 1.305 to $2.615, p<0.001$ ), and significant associations were observed in all subgroups. The PAPP-A level was also significantly higher in HDP patients than in healthy pregnant women $(\mathrm{SMD}=2.249 ; 95 \% \mathrm{Cl} 1.324$ to 3.173 , $\mathrm{p}<0.001)$. The positive association between PAPP-A level and the risk of HDP was consistently observed in all subgroups except the subgroup with low NOS score. Conclusions: The present meta-analysis suggests that an elevated PAPP-A level may be associated with susceptibilities to EH and HDP.

\section{INTRODUCTION}

As a growing public health problem in developing countries, essential hypertension $(\mathrm{EH})$ is considered a major risk factor for

\section{Strengths and limitations of this study}

- To our knowledge, the present study is the first meta-analysis focused on the association of pregnancy-associated plasma protein-A (PAPP-A) levels with essential hypertension (EH) and hypertensive disorders in pregnancy (HDP) in Chinese population.

- The results suggested that PAPP-A levels were higher in patients with EH or HDP than in healthy controls.

- Multicentre, multi-ethnicity and larger scale studies are required to clarify the actual pathogenic mechanism of PAPP-A underlying EH and HDP.

cardiovascular diseases, such as coronary heart disease, ischaemic stroke, heart failure, etc. In the last decade, the prevalence of $\mathrm{EH}$ in China has increased significantly. ${ }^{1}$ In general, the aetiology and the development of $\mathrm{EH}$ are affected by genetic as well as environmental factors. ${ }^{2}{ }^{3}$ Recently, numerous studies have shown that EH is closely related to inflammation, and inflammation plays an important role in the pathogenesis and maintenance of $\mathrm{EH}^{4}$

Hypertensive disorders in pregnancy (HDP) remain a major cause of fetal and maternal mortality. ${ }^{5}$ In general, HDP has five subtypes: (1) gestational hypertension, (2) preeclampsia, (3) eclampsia, (4) chronic hypertension in pregnancy and (5) pre-eclampsia superimposed on chronic hypertension. The true aetiology and pathophysiology of HDP remain unclear. The genetic susceptibility and other 
biophysiological factors (such as endothelial dysfunction, immune imbalance, trophoblast cell ischaemia, oxidative stress, etc) may also contribute to the pathogenesis.

Pregnancy-associated plasma protein-A (PAPP-A), first identified by Lin et af ${ }^{6}$ in 1974 , belongs to the matrix metalloproteinase family. PAPP-A is synthesised and secreted not only by placental trophoblast cells, but also by fibroblast cells, vascular smooth muscle cells, mesenchymal cells, etc. In the last decade, the role of PAPP-A in the diagnosis and prognosis of coronary heart disease has been investigated in depth. ${ }^{78}$

Several recent studies have indicated that an increased serum PAPP-A level may be a biomarker of EH and HDP based on its important effects on the proliferation of vascular smooth muscle cells, and on the reconstruction of extracellular matrix. ${ }^{9-27}$ But some results are inconsistent. $^{28} 29$ At the same time, the sample sizes of the individual studies were relatively small. Therefore, it is important to perform a meta-analysis of all available data to assess the value of peripheral blood PAPP-A levels for the diagnosis of EH and HDP.

\section{METHODS}

\section{Study selection}

The current study was conducted according to the Meta-analysis of Observational Studies in Epidemiology (MOOSE) ${ }^{30}$ group and the Preferred Reporting Items for Systematic reviews and Meta-Analyses (PRISMA) guidelines. ${ }^{31}$

Studies without language restriction, published prior to 1 December 2014, were searched from the following electronic databases: PubMed, Embase, Cochrane Library, Chinese Biomedical Database (CBM), Wanfang databases and China National Knowledge Infrastructure (CNKI). The following keywords and Medical Subject Headings (MeSH) terms were used: ('PAPP-A' or 'pregnancy associated plasma protein-A') and ('hypertension' or 'essential hypertension' or 'high blood pressure' or 'hypertension in pregnancy' or 'hypertensive disorders in pregnancy' or 'pregnancy-induced hypertension syndrome' or 'hypertensive disorder complicating pregnancy' or 'preeclampsia' or 'eclampsia' or 'gestational hypertension'). The literature in Chinese was searched using the above terms in Chinese. For example, the full search strategy used in the CNKI database is: Subject term='PAPP-A'+'pregnancy associated plasma protein-A' and (Subject term='essential hypertension'+'hypertensive disorders in pregnancy' or Keyword='hypertension in pregnancy'+'eclampsia'+'hypertensive disorder complicating pregnancy'+'gestational hypertension'+'hypertension'). A manual search was conducted by checking the references of the relevant studies to reduce omissions.

\section{Inclusion criteria}

The included studies were required to meet all the following criteria: (1) the study design had to be a casecontrol or a cohort study; (2) the study had to be used to evaluate the relationships between PAPP-A and EH or HDP; (3) the diagnostic criteria of EH was based on the criteria of WHO/International Society of Hypertension (1999 or 2005); HDP was defined as high blood pressure that was detected after a minimum of 20 weeks of pregnancy; (4) the study was conducted in Chinese population; (5) the specimen was serum or plasma, rather than placental tissue; (6) the mean and SD were clear in the case and the control groups. If the results of one population were published in multiple studies, we selected the most comprehensive study. If we had doubts about the data, we contacted the corresponding author by email.

\section{Data extraction}

Two investigators (GC and BZ) independently evaluated the eligibility of all retrieved studies and extracted the original information, including the first author's name, year of publication, average age, type of disease, geographic distribution, sample size of cases and controls, peripheral blood PAPP-A level, detecting methods and specimen type. Any disagreement was resolved by discussion or in consultation with another author (GS).

\section{Quality assessment}

A quality score was used to assess the quality of the studies according to the Newcastle-Ottawa Scale (NOS) criteria, ${ }^{32}$ including subject selection (0-4), comparability (0-2) and exposure (0-3). The quality of a paper was considered as high if the NOS scores were above $7 .^{33}$

\section{Statistics and analyses}

Because the unit weights of PAPP-A level in each study were not consistent, the standardised mean difference (SMD) with $95 \%$ CI was used to estimate the size of the effect in the present study.

Heterogeneity across studies was calculated by using Cochran's $\chi^{2}$ based Q-test. At the same time, it was also estimated by using the $\mathrm{I}^{2}$ test. If the heterogeneity was statistically significant $\left(\mathrm{p}<0.01, \quad \mathrm{I}^{2}>50 \%\right)$, the random-effects model (a DerSimonian-Laird method) was used. Otherwise, the fixed-effects model (a MantelHaenszel method) was applied. ${ }^{34}$ To identify the sources of heterogeneity, subgroup analyses were performed. The studies were stratified by sample size $(>100$ or $\leq 100$ ), specimen type (serum or plasma), detecting method (ELISA or Chemiluminescence), NOS score $(\geq 7$ or $<7$ ), type of disease (EH or HDP) and geographical location (North China or South China). A metaregression analysis was also performed to identify sources of heterogeneity among studies. To assess the stability of results, sensitivity analysis was used by calculating the pooled effect again when omitting a single study, sequentially.

The publication bias was assessed via Begg's funnel plot and Egger's regression test. If the funnel plot was asymmetric, the trim-and-fill method was used to identify and correct the publication bias. 
The STATA V.12.0 (StataCorp LP, College Station, Texas, USA 77845 USA) was used for the statistical analysis. A $p$ value $<0.05$ was considered statistically significant.

\section{RESULTS}

\section{Study selection}

A total of 214 potentially pertinent articles were identified, using the above search strategy. One hundred and seventy-three articles were excluded after the title and abstract review. Another 21 studies were excluded after we reviewed the full article texts. Finally, a total of 20 studies involving 1493 patients and 1839 controls met the inclusion criteria and were included for the current meta-analysis. The detailed process of the literature search and study selection is described in figure 1.

\section{Study characteristics}

Among the 20 studies, six studies involving 395 cases and 233 controls were focused on the relationship between the level of PAPP-A and EH, and 14 studies involving 1098 cases and 1606 controls were focused on PAPP-A and HDP. The characteristics of the 20 studies are summarised in table 1 . All of the 20 studies were published in Chinese. Publication years of the eligible studies ranged from 2005 to 2014 . The sample size of each study varied from 80 to 814 . Nineteen studies were conducted in Han population and one study was conducted in Kazakh population. Seventeen studies used serum specimens to examine the PAPP-A level, and three studies used plasma. Nineteen studies, except Zhu's, used ELISA as a method to detect PAPP-A level. ${ }^{27}$ The NOS scores in 17 of the 20 studies were above seven.

\section{Association between the PAPP-A level and EH}

The pooled SMD was calculated by random-effects model because there was significant heterogeneity between studies $\left(\mathrm{I}^{2}=89.7 \%, \mathrm{p}<0.001\right)$. As shown in figure 2, the pooled analysis indicated that the PAPP-A level was significantly higher in $\mathrm{EH}$ patients than in controls $(\mathrm{SMD}=1.960,95 \%$ CI 1.305 to $2.615, \mathrm{p}<0.001)$.

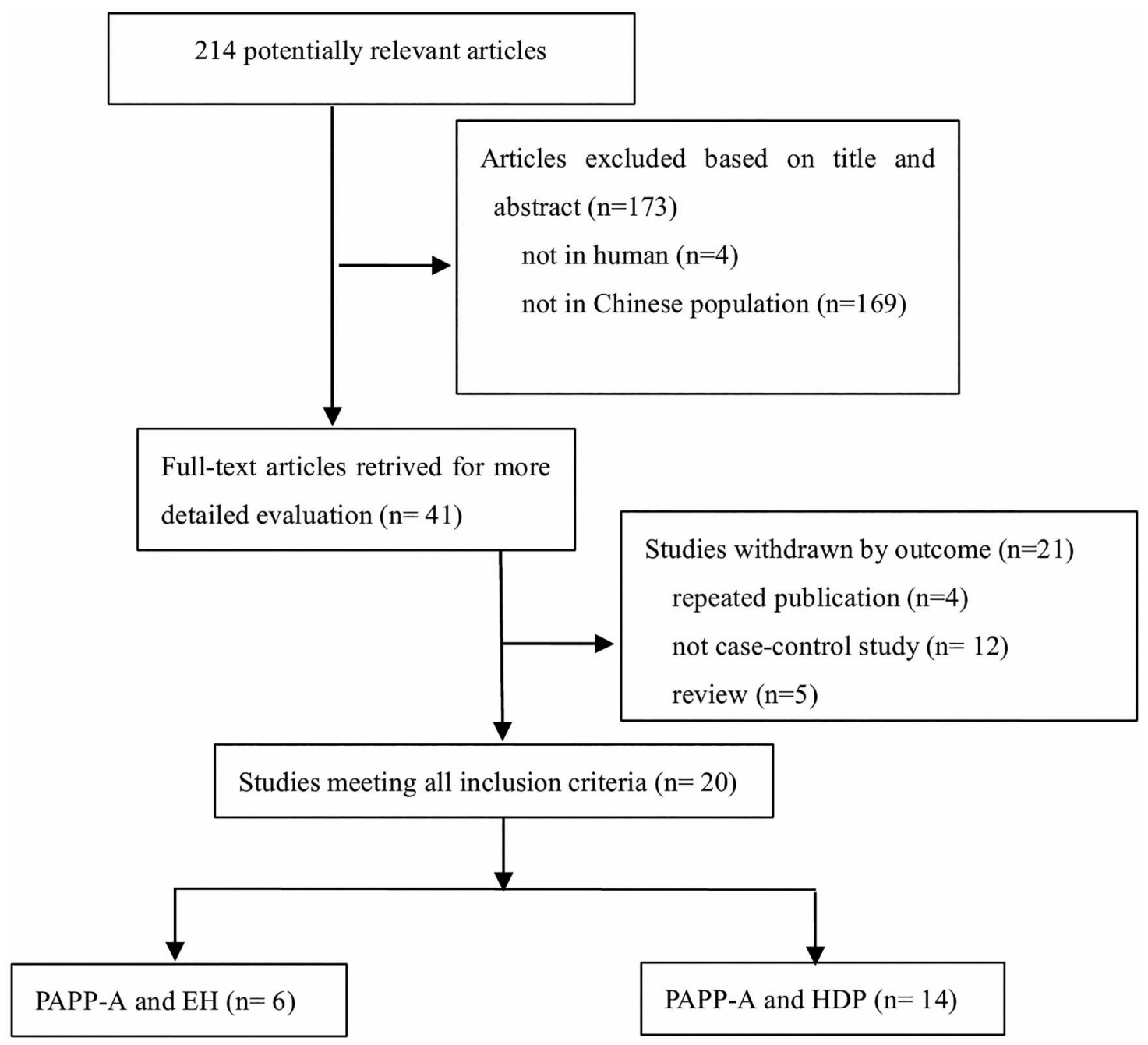

Figure 1 Flow diagram of article selection process. EH. EH, essential hypertension; HDP, hypertensive disorders in pregnancy; PAPP-A, pregnancy-associated plasma protein-A. 
Table 1 Characteristics of 20 studies on PAPP-A and risk of EH and HDP

\begin{tabular}{|c|c|c|c|c|c|c|c|c|c|c|c|c|c|c|}
\hline \multirow[b]{2}{*}{ Author } & \multirow[b]{2}{*}{ Year } & \multirow{2}{*}{$\begin{array}{l}\text { Type of } \\
\text { disease }\end{array}$} & \multirow{2}{*}{$\begin{array}{l}\text { Geographic } \\
\text { distribution }\end{array}$} & \multirow[b]{2}{*}{ Age (case/control) } & \multicolumn{3}{|c|}{ Case } & \multicolumn{3}{|c|}{ Control } & \multirow{2}{*}{$\begin{array}{l}\text { Detecting } \\
\text { method }\end{array}$} & \multirow[b]{2}{*}{ Unit } & \multirow{2}{*}{$\begin{array}{l}\text { Specimen } \\
\text { type }\end{array}$} & \multirow[b]{2}{*}{ NOS } \\
\hline & & & & & $\mathbf{N}$ & $\mathbf{M}$ & SD & $\mathbf{N}$ & $\mathbf{M}$ & SD & & & & \\
\hline $\mathrm{Li} \mathrm{CF}^{9}$ & 2005 & HDP & Shanxi & $26.5 \pm 4.3 /-$ & 60 & 58.85 & 21.05 & 60 & 36.8 & 10.4 & ELISA & IU/L & serum & 8 \\
\hline Xia JX $X^{10}$ & 2005 & HDP & Guangdong & $27.85 \pm 5.20 / 27.50 \pm 5.47$ & 95 & 46.36 & 14.83 & 103 & 23.6 & 6.1 & ELISA & $\mathrm{mIU} / \mathrm{mL}$ & serum & 8 \\
\hline Wang $L Y^{11}$ & 2007 & $\mathrm{EH}$ & Jilin & $-/ 60 \pm 12$ & 70 & 3 & 0.6 & 56 & 1.5 & 0.5 & ELISA & $\mathrm{mU} / \mathrm{L}$ & serum & 6 \\
\hline Zhou Z Z $^{12}$ & 2007 & HDP & Jiangsu & $5.8 \pm 4.8 / 26.5 \pm 4.3$ & 109 & 57.84 & 12.25 & 105 & 29.4 & 9.65 & ELISA & $\mathrm{mg} / \mathrm{L}$ & serum & 8 \\
\hline Fan $\mathrm{JF}^{13}$ & 2009 & $\mathrm{EH}$ & Anhui & $64.19 \pm 13.5 / 61.78 \pm 16.9$ & 84 & 8.66 & 3.26 & 22 & 5.17 & 3.15 & ELISA & $\mathrm{mIU} / \mathrm{L}$ & serum & 6 \\
\hline Liu JG ${ }^{22}$ & 2010 & HDP & Hunan & $29.7 \pm 5.7 / 30.2 \pm 4.1$ & 40 & 2.61 & 0.96 & 40 & 1.83 & 0.75 & ELISA & $\mu \mathrm{g} / \mathrm{L}$ & serum & 8 \\
\hline Pan $Q^{14}$ & 2010 & HDP & Jiangsu & $26.8 \pm 4.7 / 26.3 \pm 3.8$ & 92 & 58.34 & 12.19 & 35 & 28.4 & 8.57 & ELISA & $\mathrm{U} / \mathrm{L}$ & serum & 8 \\
\hline Wang $\mathrm{J}^{15}$ & 2010 & $\mathrm{EH}$ & Anhui & $64 \pm 12 / 61 \pm 16$ & 80 & 11.91 & 3.42 & 15 & 7.1 & 1.9 & ELISA & $\mathrm{mU} / \mathrm{L}$ & serum & 6 \\
\hline Zhang $\mathrm{JY}^{16}$ & 2010 & HDP & Hebei & $23-36 / 22-35$ & 115 & 181 & 32 & 127 & 69 & 5 & ELISA & $\mathrm{mU} / \mathrm{mL}$ & serum & 6 \\
\hline Meng $\mathrm{ZH}^{17}$ & 2011 & $\mathrm{EH}$ & Xinjiang & $56.6 \pm 11.7 / 56.4 \pm 13.2$ & 80 & 6.04 & 1.07 & 30 & 3.9 & 0.64 & ELISA & $\mathrm{mg} / \mathrm{L}$ & plasma & 9 \\
\hline $\mathrm{Xu} \mathrm{XL^{18 }}$ & 2011 & HDP & Zhejiang & $25.7 \pm 5.7 /-$ & 70 & 58.8 & 12.47 & 70 & 30.5 & 10.5 & ELISA & IU/L & serum & 8 \\
\hline Zhang $C Y^{19}$ & 2011 & $\mathrm{EH}$ & Shandong & $61 \pm 9 / 52 \pm 13$ & 39 & 21.22 & 4.12 & 64 & 7.97 & 4.36 & ELISA & $\mathrm{mU} / \mathrm{L}$ & plasma & 5 \\
\hline Cui FL 20 & 2012 & HDP & Yunnan & $27.3 \pm 2.4 / 27.1 \pm 2.6$ & 72 & 51.2 & 10.4 & 75 & 32.7 & 9.6 & ELISA & $\mathrm{IU} / \mathrm{L}$ & serum & 8 \\
\hline Gao L $L^{21}$ & 2012 & HDP & Sichuan & $32.49 \pm 2.1 / 29.15 \pm 2.3$ & 120 & 73.39 & 5.33 & 40 & 38.2 & 4.56 & ELISA & $\mathrm{U} / \mathrm{L}$ & serum & 8 \\
\hline $\mathrm{Hu} \mathrm{Y} \mathrm{Y}^{28}$ & 2012 & HDP & Henan & $-1-$ & 88 & 19.98 & 5.36 & 726 & 22.5 & 1.62 & ELISA & $\mathrm{U} / \mathrm{L}$ & serum & 5 \\
\hline Peng $\mathrm{HY}^{23}$ & 2012 & HDP & Guangdong & $8.6 \pm 7.7 / 28.8 \pm 7.6$ & 45 & 56.7 & 12.6 & 45 & 29.1 & 12.2 & ELISA & $\mathrm{mU} / \mathrm{L}$ & serum & 8 \\
\hline Zhong $\mathrm{CX}^{24}$ & 2012 & HDP & Guangdong & $27 \pm 4.2 / 28.2 \pm 4.0$ & 50 & 2.6 & 0.94 & 50 & 1.84 & 0.73 & ELISA & $\mu \mathrm{g} / \mathrm{L}$ & serum & 7 \\
\hline Liu $Z^{25}$ & 2014 & HDP & Jilin & $28.27 \pm 3.00 / 27.13 \pm 2.80$ & 50 & 62.7 & 11.7 & 50 & 28.2 & 13.1 & ELISA & $\mathrm{mIU} / \mathrm{L}$ & serum & 8 \\
\hline Zhang $\mathrm{L}^{26}$ & 2014 & $\mathrm{EH}$ & Guangdong & $37 \pm 8 / 35 \pm 9$ & 42 & 65.7 & 16.5 & 46 & 48.8 & 10.4 & ELISA & $\mu \mathrm{g} / \mathrm{L}$ & plasma & 8 \\
\hline Zhu $\mathrm{F}^{27}$ & 2014 & HDP & Jiangsu & $27.5 \pm 5.3 / 26.2 \pm 4.5$ & 92 & 63.06 & 21.98 & 80 & 40.3 & 11.5 & $\begin{array}{l}\text { Chemilumine- } \\
\text { scence }\end{array}$ & U/L & serum & 7 \\
\hline
\end{tabular}




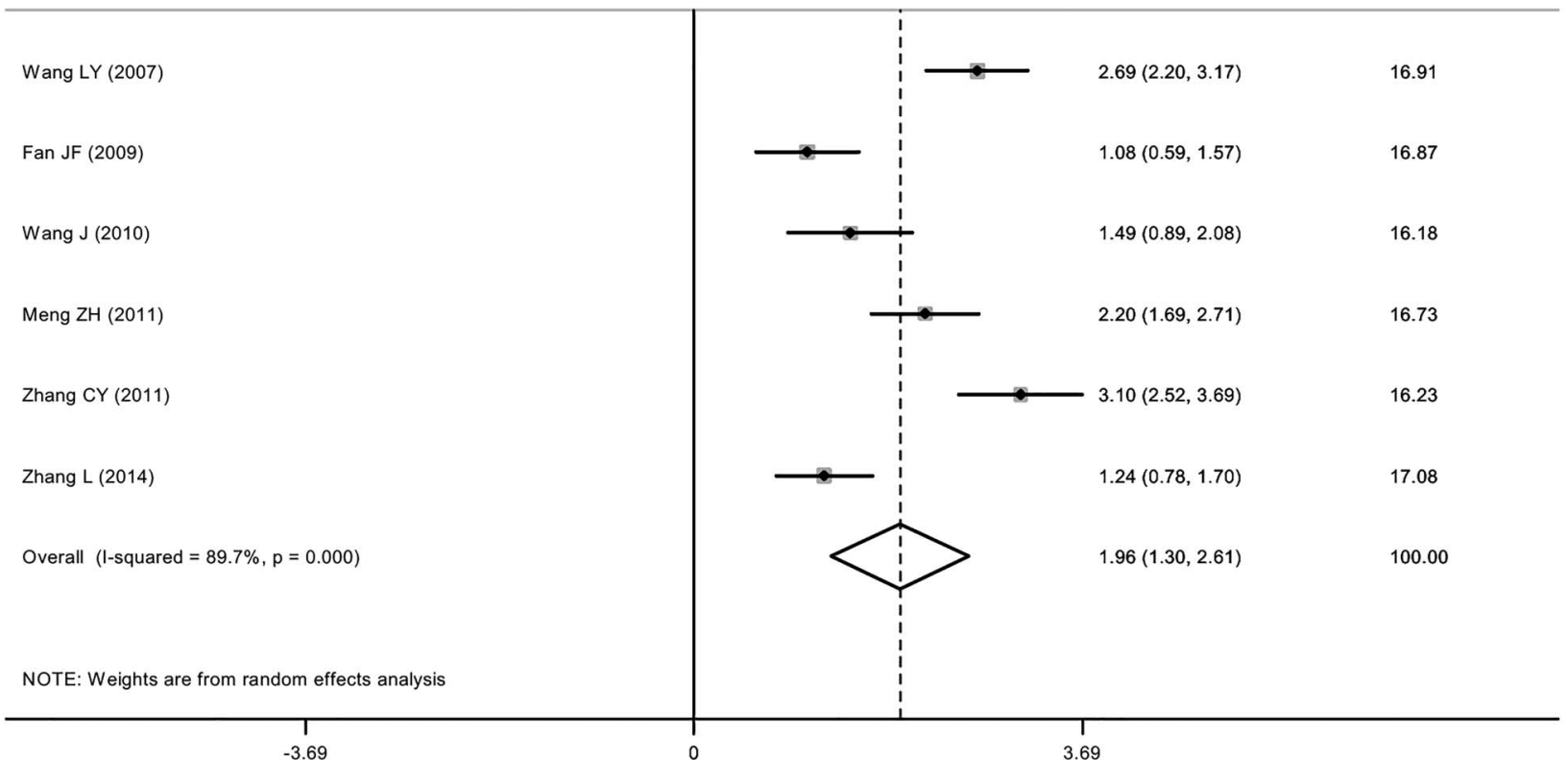

Figure 2 Forest plot of relationship between the level of PAPP-A and EH. EH, essential hypertension; PAPP-A, pregnancyassociated plasma protein-A; SMD, standardised mean difference.

In the stratified analyses based on sample size, specimen type, detecting methods, NOS score and geographical location, significant associations were observed in all the subgroups (table 2).

The stability of the study was detected by sensitivity analysis, through re-meta-analysis, by excluding one individual study in sequence. As shown in figure 3, no individual study significantly affects the pooled SMD.
The publication bias was assessed by Begg's funnel plot and Egger's test. The funnel plot was symmetric and the Egger's test also suggested no publication bias ( $\mathrm{p}=0.450)$ (figure 4$)$.

\section{Association between the PAPP-A level and HDP}

An obvious heterogeneity was found $\left(\mathrm{I}^{2}=98.6 \%\right.$, $\mathrm{p}<0.001)$, so the pooled SMD was also calculated by

Table 2 Effect estimates in subgroup analyses

\begin{tabular}{|c|c|c|c|c|c|c|c|c|}
\hline \multirow{2}{*}{$\begin{array}{l}\text { Subgroup } \\
\text { according to }\end{array}$} & \multicolumn{4}{|c|}{ EH } & \multicolumn{4}{|c|}{ HDP } \\
\hline & $\mathbf{N}$ & SMD $(95 \% \mathrm{Cl})$ & $I^{2}(\%)$ & $\mathbf{P}_{\text {heterogeneity }}$ & $\overline{\mathbf{N}}$ & SMD (95\% Cl) & $I^{2}(\%)$ & $\mathbf{P}_{\text {heterogeneity }}$ \\
\hline Total & 6 & 1.960 (1.305 to 2.615$)$ & 89.7 & $<0.0001$ & 14 & 2.249 (1.324 to 3.173$)$ & 98.6 & $<0.0001$ \\
\hline \multicolumn{9}{|l|}{ NOS score } \\
\hline$\geq 7$ & 2 & 1.711 (0.770 to 2.652$)$ & 86.7 & 0.006 & 12 & 2.270 (1.694 to 2.846 ) & 95.3 & $<0.0001$ \\
\hline$<7$ & 4 & 2.086 (1.140 to 3.033$)$ & 92.0 & $<0.0001$ & 2 & $1.962(-4.005$ to 7.929$)$ & 99.8 & $<0.0001$ \\
\hline \multicolumn{9}{|l|}{ Specimen type } \\
\hline Serum & 3 & 1.755 (0.752 to 2.757$)$ & 91.0 & 0.001 & 14 & 2.249 (1.324 to 3.173$)$ & 98.6 & $<0.0001$ \\
\hline Plasma & 3 & 2.167 (1.117 to 3.217$)$ & 92.0 & $<0.0001$ & 0 & - & - & \\
\hline \multicolumn{9}{|l|}{ Sample size } \\
\hline$>100$ & 3 & 1.988 (1.042 to 2.935$)$ & 90.8 & $<0.0001$ & 10 & 2.472 (1.254 to 3.691 ) & 99.0 & $<0.0001$ \\
\hline$\leq 100$ & 3 & 1.935 (0.807 to 3.061$)$ & 92.3 & $<0.0001$ & 4 & 1.690 (0.783 to 2.597$)$ & 92.9 & $<0.0001$ \\
\hline \multicolumn{9}{|l|}{ Geographical location } \\
\hline North China & 3 & 2.649 (2.155 to 3.142$)$ & 62.2 & 0.071 & 10 & 3.969 (1.720 to 6.218$)$ & 98.6 & $<0.0001$ \\
\hline South China & 3 & 1.242 (0.950 to 1.533$)$ & 0 & 0.582 & 4 & 1.574 (0.649 to 2.500$)$ & 98.4 & $<0.0001$ \\
\hline \multicolumn{9}{|l|}{ Detecting methods } \\
\hline ELISA & 6 & 1.960 (1.305 to 2.615$)$ & 89.7 & $<0.0001$ & 13 & 2.327 (1.307 to 3.346$)$ & 98.7 & $<0.0001$ \\
\hline Chemiluminescence & 0 & - & - & - & 1 & $1.272(0.943$ to 1.601$)$ & 0 & \\
\hline
\end{tabular}


Figure 3 Analysis of influence of individual study on the pooled estimate in $\mathrm{EH}$ group (open circles indicate the pooled SMD. Horizontal lines represent the 95\% Cls). EH, essential hypertension; SMD, standardised mean difference.
Meta-analysis estimates, given named study is omitted

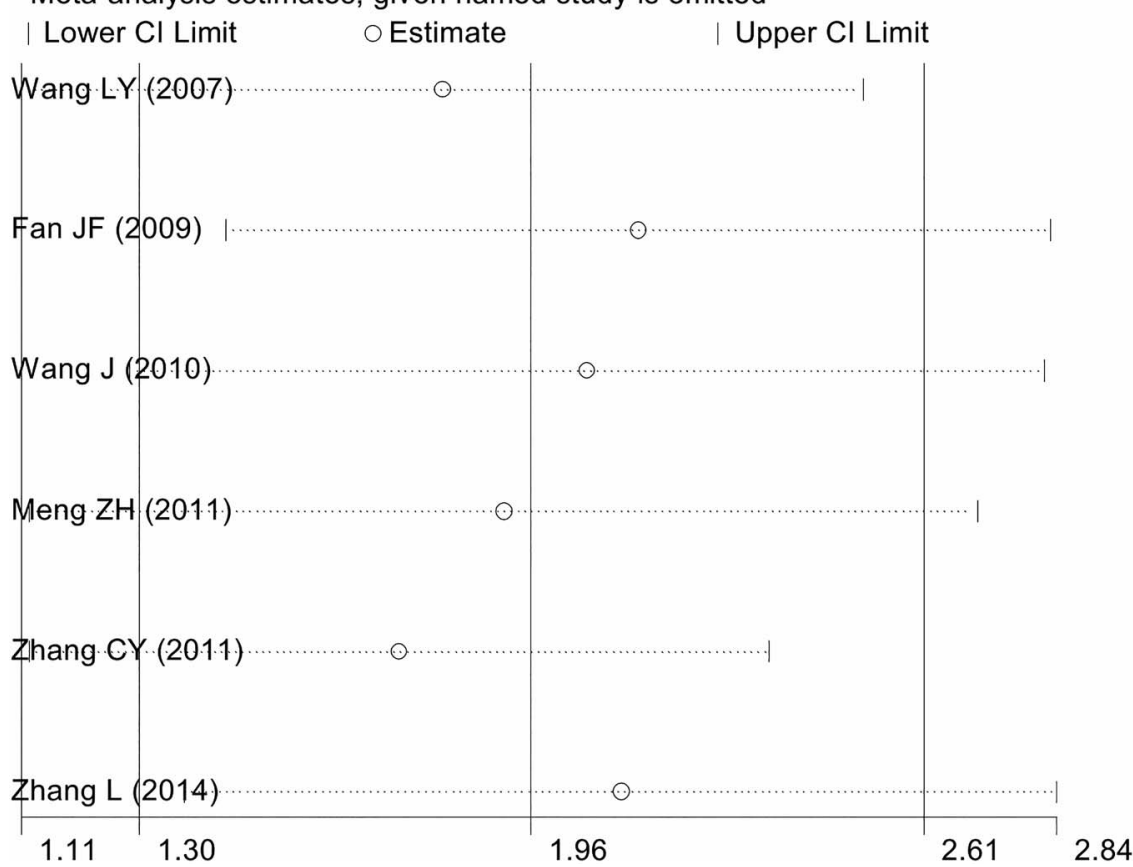

random-effects model. As shown in figure 5, the results have demonstrated that the PAPP-A level was significantly higher in HDP patients than in healthy pregnant women (SMD=2.249; 95\% CI 1.324 to 3.173 , $\mathrm{p}<0.001$ ).

Stratified analyses were conducted based on sample size, detecting methods, NOS score and geographical location. A positive association between PAPP-A level and the risk of HDP was consistently observed in all subgroups except the subgroup with low NOS score (table 2).

The sensitivity analysis suggested that only the $\mathrm{Hu} \mathrm{Y}^{28}$ study appeared to have a large influence, but the result did not change after this study was excluded, suggesting that the results have sufficient statistical power (figure 6).

Owing to the significant heterogeneity between studies, besides the subgroup analyses, a meta-regression was also

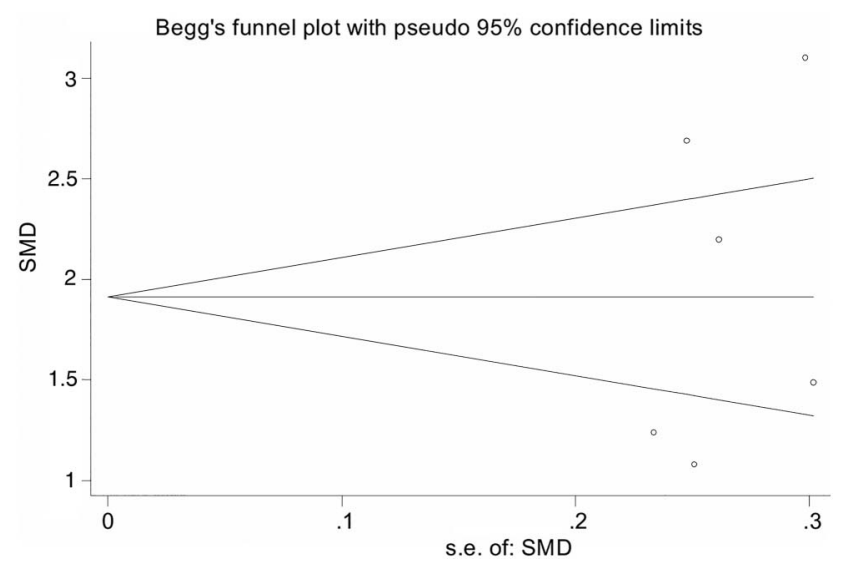

Figure 4 Funnel plot of PAPP-A associated with EH (each point represents a separate study for the indicated association). $\mathrm{EH}$, essential hypertension; PAPP-A, pregnancy-associated plasma protein-A; SMD, standardised mean difference. performed to further identify the potential source of heterogeneity. Sample size, detecting methods, NOS score and geographical location were included in regression analysis. The result indicated that only geographical location might partially contribute to the heterogeneity $\left(\mathrm{P}_{\text {meta-regression }}=0.025\right)$ and can explain $30.61 \%$ of the heterogeneity. Other factors, such as NOS score $\left(\mathrm{P}_{\text {meta-regression }}=0.812\right)$, sample size $\left(\mathrm{P}_{\text {meta-regression }}=0.507\right)$ and detecting methods $\left(\mathrm{P}_{\text {meta-regression }}=0.605\right)$, might not be sources of heterogeneity.

The obvious asymmetry of the funnel plot was visualised (Egger's test $\mathrm{p}=0.000$ ) (figure 7). The trim-and-fill method was used to identify and correct publication bias. ${ }^{35}$ The number of missing studies was estimated by the linear method. After two iterations, the value of difference became 0 and the number of estimated deficient study was 0 , indicating that the result was stable.

\section{DISCUSSION}

To our knowledge, the present study is the first meta-analysis focused on the association of PAPP-A levels with EH and HDP. We found that PAPP-A levels were higher in patients with EH or HDP than in healthy controls.

In 2011, a multivariable study of large-scale samples, involving 112386 pregnant women, was conducted to estimate the prevalence of HDP in China. ${ }^{36}$ This epidemiological investigation revealed that the prevalence of HDP in China is about $5.22 \%$, though smaller than $8-10 \%$, as seen in other studies. ${ }^{37}$ Although numerous studies have been conducted, the precise mechanism underlying the pathogenesis of $\mathrm{EH}$ and HDP remains poorly understood.

PAPP-A, about $750 \sim 820 \mathrm{kDa}$ in size, plays an important role in the proliferation of vascular smooth muscle 


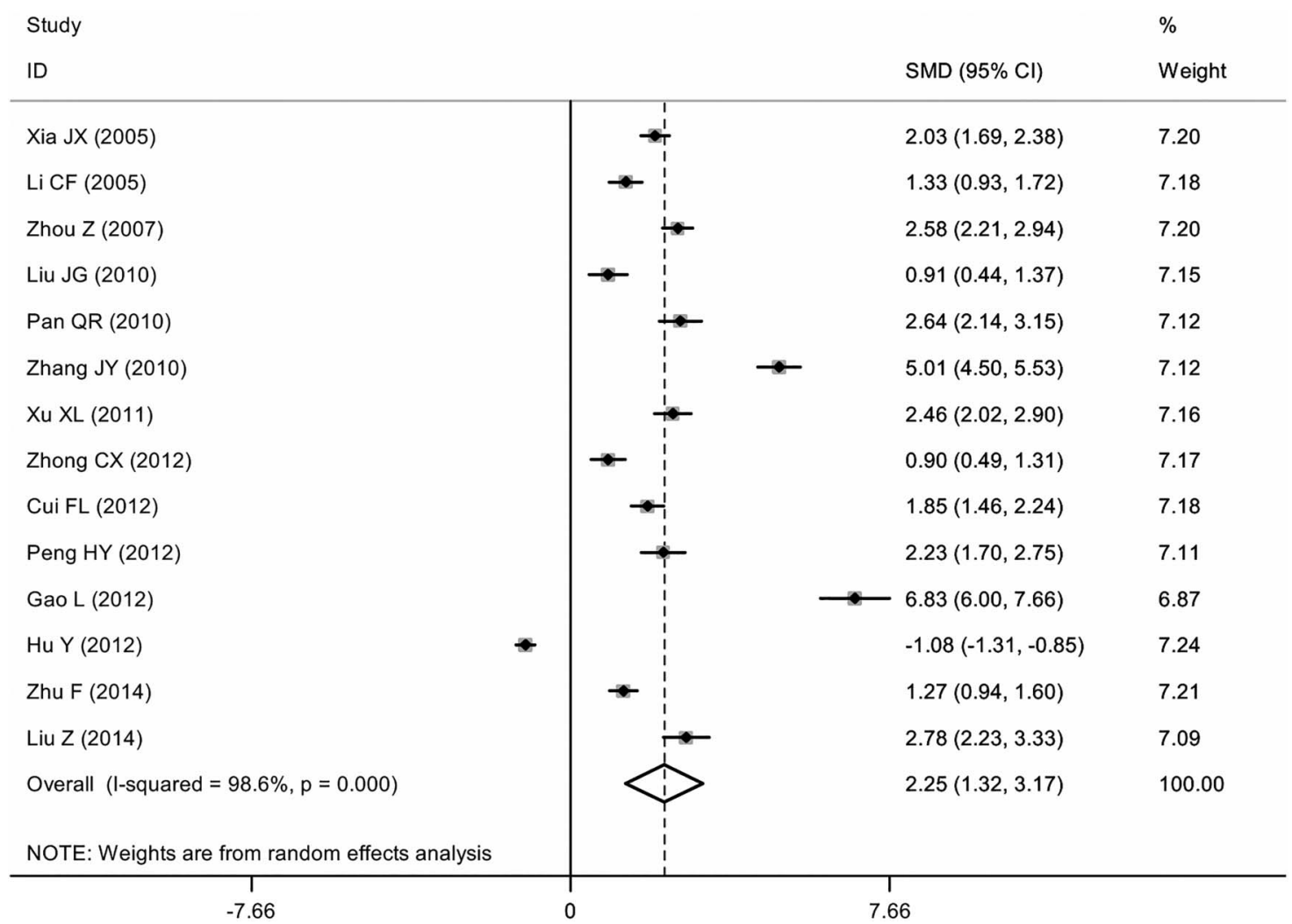

Figure 5 Forest plot of relationship between the level of PAPP-A and HDP. HDP, hypertensive disorders in pregnancy; PAPP-A, pregnancy-associated plasma protein-A; SMD, standardised mean difference.

cells and in the reconstruction of the extracellular matrix. Previous studies have indicated that the low serum PAPP-A concentrations in pregnant women were associated with adverse pregnancy outcomes, such as premature delivery and small for gestational age infants, etc. $^{38}$ Recently, a growing number of studies have suggested elevated serum level of PAPP-A might be involved in the development of EH and HDP by the following mechanisms: (1) PAPP-A may increase the IGF-1 level, which can promote the proliferation of vascular smooth muscle cells and remodelling of the extracellular matrix; (2) The elevated IGF-I level also increases
Figure 6 Analysis of influence of individual study on the pooled estimate in HDP group (open circles indicate the pooled SMD. Horizontal lines represent the 95\% Cls). HDP, hypertensive disorders in pregnancy; SMD, standardised mean difference.
Meta-analysis estimates, given named study is omitted

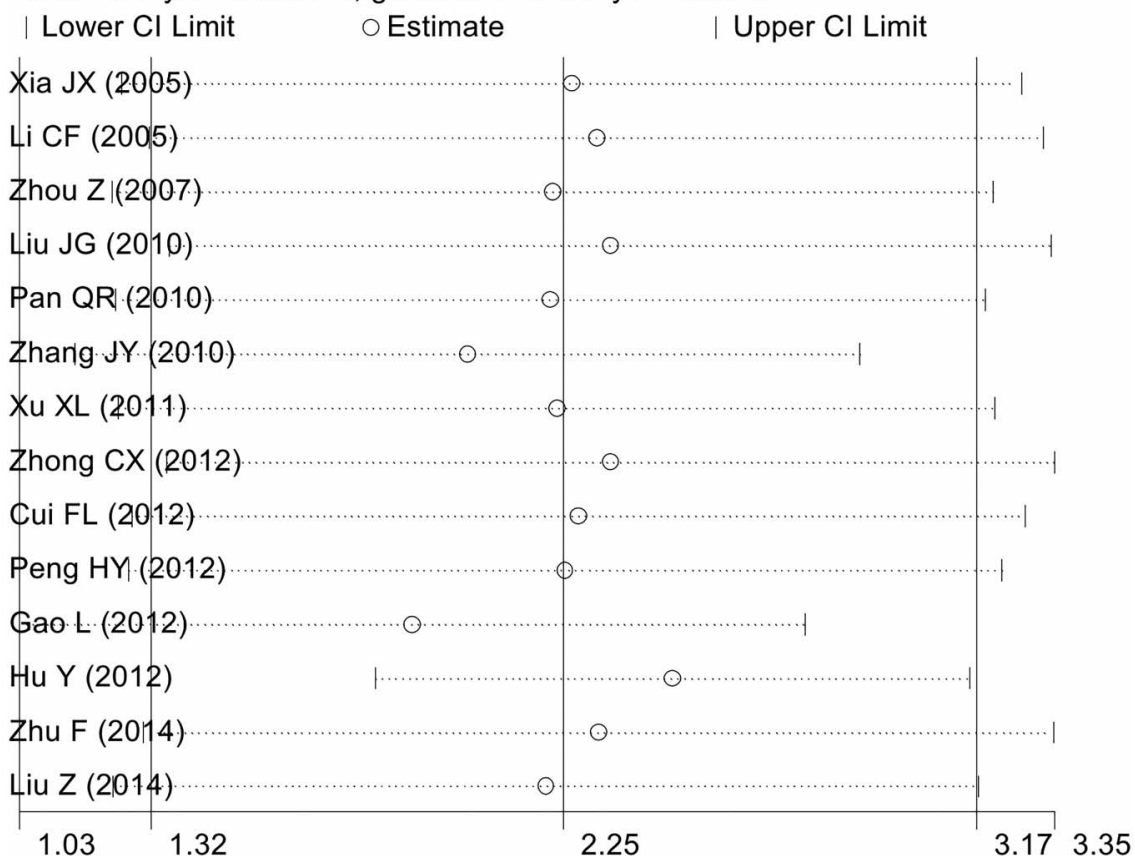




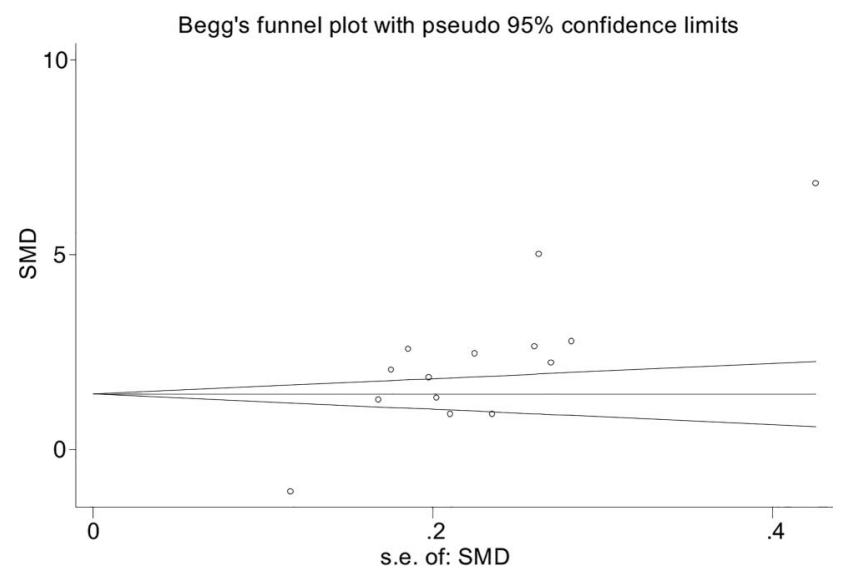

Figure 7 Funnel plot of PAPP-A associated with HDP (each point represents a separate study for the indicated association). HDP, hypertensive disorders in pregnancy; PAPP-A, pregnancy-associated plasma protein-A; SMD, standardised mean difference.

the degradation of the NO synthetase, which can lead to a sustained contraction of vessel wall; (3) PAPP-A can promote the release of inflammatory cytokines, which can increase arterial endothelial injury; (4) In HDP patients, hypoxia causes the trophoblast cells to secrete more PAPP-A, which can augment placenta local oxidative stress and aggravate the placenta hypoxia.

Although most of the studies reached positive conclusions, the results were not consistent and the sample sizes in each study were relatively small. To resolve this problem, we conducted the current meta-analysis to examine the association of PAPP-A levels with risk of $\mathrm{EH}$ and HDP.

In the present meta-analysis, 20 studies met the inclusion criteria. Six were focused on $\mathrm{EH}$ and 14 were focused on HDP. According to the results, we found that the PAPP-A level was significantly higher in EH $(\mathrm{SMD}=1.960,95 \%$ CI 1.305 to $2.615, \mathrm{p}<0.001)$ and HDP patients $(\mathrm{SMD}=2.249 ; 95 \%$ CI 1.324 to $3.173, \mathrm{p}<0.001)$ than in controls.

A significant heterogeneity was found among studies. A meta-regression was performed to identify the sources of heterogeneity in the HDP group. We found that only the geographical location can explain $30.61 \%$ of the heterogeneity. In the previous studies, the prevalence of $\mathrm{EH}$ and HDP in Chinese population represents a characteristic of geographical location, which is higher in North China than in South China. Although the exact mechanism is not clear, cold-induced vasoconstriction might trigger the onset of these diseases. Our meta-analysis suggested that geographical location might be a source of heterogeneity. Subgroup analyses were also performed in the EH and HDP groups. The stratified analyses were conducted according to sample size, detecting methods, NOS score and geographical location. The positive association of PAPP-A levels with EH and HDP was consistently observed in all of the subgroups except the subgroup with low NOS score. We also performed a sensitivity analysis to assess the influence of individual studies on the pooled SMD. No single study influenced the stability of the whole study.

Although our meta-analysis is a practical way to generate a more powerful result with less random error than each individual study, our study has some limitations. First, the total sample sizes of the studies included in this meta-analysis were relatively small. Second, all participants in the studies came from hospitals, which may lead to selection bias. Third, research has revealed that PAPP-A levels vary in different subtypes of the disease. The PAPP-A level is different even between early-onset pre-eclampsia and late-onset preeclampsia. $^{28}$ At the same time, it is increasingly accepted that early-onset pre-eclampsia and late-onset pre-eclampsia have different pathogenic mechanisms. ${ }^{39}$ However, some studies included in our analysis were not divided into subgroups. Fourth, blood collection time was not noted in the studies, which could contribute to the heterogeneity. Fifth, obvious publication bias was found in the HDP group: it is more difficult to publish negative results and also, the study population was limited to the Chinese population. Although obvious publication bias exists, sensitivity analysis of the trim-and-fill method showed the result was reliable. Finally, a major confounding factor was the heterogeneity among studies. In the $\mathrm{EH}$ group, as the number of studies was smaller than 10, the meta-regression method was not repeated, so further identification of the source of heterogeneity was not performed. In the HDP group, although meta-regression was performed, only part of the heterogeneity could be explained by geographical location.

\section{CONCLUSIONS}

In conclusion, our meta-analysis suggests that PAPP-A levels are significantly higher in $\mathrm{EH}$ and HDP patients than in controls. However, multicentre, multiethnicity and larger-scale studies are required to clarify the actual pathogenic mechanism of PAPP-A underlying EH and HDP.

\section{Author affiliations}

${ }^{1}$ Department of Cardiology, Wujin Hospital, Affiliated To Jiangsu University, Changzhou, Jiangsu Province, China

${ }^{2}$ Department of Pathology and Molecular Medicine, McMaster University, Hamilton, Ontario, Canada

${ }^{3}$ Jiangsu University Health Science Center, Zhenjiang, Jiangsu Province, China

Acknowledgements The authors are grateful to the staff of the department of Cardiology, Wujin Hospital.

Contributors $\mathrm{GC}$ and $\mathrm{BZ}$ conceived and designed the experiments. GC, BZ, WW, LY, GS, SX and XF performed the experiments. GC, BZ and XF analysed the data. GC and BZ wrote the paper: GC, BZ and XF contributed reagents/ materials/analysis tools.

Competing interests None declared.

Provenance and peer review Not commissioned; externally peer reviewed.

Data sharing statement No additional data are available. 
Open Access This is an Open Access article distributed in accordance with the Creative Commons Attribution Non Commercial (CC BY-NC 4.0) license, which permits others to distribute, remix, adapt, build upon this work noncommercially, and license their derivative works on different terms, provided the original work is properly cited and the use is non-commercial. See: http:// creativecommons.org/licenses/by-nc/4.0/

\section{REFERENCES}

1. Wang X, Bots ML, Yang F, et al. Prevalence of hypertension in China: a systematic review and meta-regression analysis of trends and regional differences. J Hypertens 2014;32:1919-27.

2. Cai G, Zhang B, Weng W, et al. E-Selectin gene polymorphisms and essential hypertension in Asian population: An Updated Meta-Analysis. PLoS ONE 2014;9:e102058.

3. Tang N, Li JW, Liu YM, et al. Human cytomegalovirus infection is associated with essential hypertension in Kazakh and Han Chinese populations. Med Sci Monit 2014;20:2508-19.

4. Bautista LE, Vera LM, Arenas IA, et al. Independent association between inflammatory markers (C-reactive protein, interleukin-6, and TNF-alpha) and essential hypertension. J Hum Hypertens 2005;19:149-54.

5. Duley L. The global impact of pre-eclampsia and eclampsia. Semin Perinatol 2009;33:130-7.

6. Lin TM, Galbert SP, Kiefer D, et al. Characterization of four human pregnancy-associated plasma proteins. Am J Obstet Gynecol 1974;118:223-36.

7. Li Y, Zhou C, Zhou X, et al. Pregnancy-associated plasma protein A predicts adverse vascular events in patients with coronary heart disease: a systematic review and meta-analysis. Arch $\mathrm{Med} \mathrm{Sci}$ 2013;9:389-97.

8. Gururajan P, Gurumurthy P, Nayar P, et al. Pregnancy associated plasma protein-A (PAPP-A) as an early marker for the diagnosis of acute coronary syndrome. Indian Heart J 2012;64:141-5.

9. Li CF, Fang J. Relationship between the levels of PAPP-A, VCAM-1 in pregnant women and hypertensive disorders in pregnancy. $J$ Pract Med 2005;21:1032-3.

10. Xia JX. Study on the relationship between the serum PAPP-A level in pregnant women and pregnancy-induced hypertension. $J$ Pract Obstet Gynecol 2005;21:187-8.

11. Wang LY, Cai GJ, Zhang WW, et al. Association between pregnancy-associated plasma protein-A and hypertension. Chin Heart J 2007; 19:74-5, 78.

12. Zhou Z. Changes of the serum PAPP-A, VCAM-1 levels in pregnancy-induced hypertension patients. Shangdong Med $J$ 2007;47:117-18.

13. Fan JF, Tang SX, Wang J, et al. Research on relationship between pregnancy associated plasma protein-A, C reactive protein and hypertension. Int J Intern Med 2009;36:12-14.

14. Pan QR. Study the significance of blood VEGF, PAPP-A, D-D and urinary NAG/Cr ratio in patients with PIH. Int $J$ Lab Med 2010;31:561-2, 564 .

15. Wang J, He SB, Tang SX, et al. Relationship between pregnancy associated plasma protein $A$ and blood pressure in patients with essential hypertension. Clin J Diffic Compl Cas 2010;9:331-3.

16. Zhang JY, Han X, Ma JH, et al. Relationship between the changes of PAPP-A, sICAM-1 and sVCAM-1and preeclampsia. Hebei Med $J$ 2010;32:672-3.

17. Meng $\mathrm{ZH}$, Wang $\mathrm{Z}$, Wang $\mathrm{L}$, et al. Changes and clinical significance of pregnancy-associated plasma protein A levels in patients with essential hypertension in Xinjiang Kazakhs. Clin Focus 2011;26:1048-50.

18. $\mathrm{Xu} \mathrm{XL}$. Study on the relationship between pregnancy associated plasma protein-A and hypertensive disorders in pregnancy. J Qiqihar Med Coll 2011;32:26-7.

19. Zhang CY, Tian YF, Ma CB, et al. Study of correlations among plasma pregnancy associated plasma protein-A relevant factors in hypertension patients with impaired glucose regulation. J Taishan Med coll 2011;32:569-72.

20. Cui FL. Expression of vascular cell adhesion molecule-1 and pregnancy associated plasma protein-A in the serum of patients with hypertensive disorders in pregnancy. Chinese $J$ Ethnomed Ethnopharm 2012;21:62-3.

21. Gao L, Chen $\mathrm{Y}$, Liang $\mathrm{H}$, et al. Expression of VCAM-1 and PAPP-A in hypertensive disorders of pregnancy and their correlation. J Pract Obstet Gynecol 2012;28:377-80.

22. Liu JG, Sun CX, Liu LL, et al. Research on the relationship between the serum VEGF, PAPP-A levels in pregnant women and the onset of hypertensive disorders in pregnancy. Pract Clin Med 2010;11:3-5.

23. Peng HY, Hu CL. Study on correlation between PAPPA and pregnancy induced hypertension. J Chengdu Med Coll 2012;7:408-9.

24. Zhong CX, Gu LH, Huang BM. The correlation between maternal serum VEGF and PAPP-A levels and the incidence of gestational hypertension. Guide China Medcine 2012;10:40-1.

25. Liu Z. Research in preeclampsia combined detection of PAPP-A and ADAM12 and its clinical significance [Master]. Jilin University, 2014.

26. Zhang L, Li J, Wu ZP. Analysis of the relationship between pregnancy associated plasma protein A and matrix metalloproteinases 9 in young and middle-aged patients and coronary heart disease. Clin Focus 2014;29:59-60.

27. Zhu F, Zhou L, Liu JB, et al. Levels and pathophysiological roles of soluble vascular cell adhesion molecule-l, pregnancy associated plasma protein A and interleukin-6 in pregnancy-induced hypertension. Med J Chin PAPF 2014;25:886-8.

28. Hu Y. Studies on correlation of pregnancy-associated plasma protein $A$ and pathological changes of placenta in patients with pregnanyc-induced hypertension. China Mod Doct 2012;50: 43-4, 46.

29. Ceylan N, Ozaksit G, Unlu BS, et al. Can first trimester placental protein-13 and pregnancy-associated plasma protein-A predict pre-eclampsia in Turkish women? J Obstet Gynaecol 2014;34:482-5.

30. Stroup DF, Berlin JA, Morton SC, et al. Meta-analysis of observational studies in epidemiology: a proposal for reporting Meta-analysis of observational studies in epidemiology (MOOSE) group. JAMA 2000;283:2008-12.

31. Moher D, Liberati A, Tetzlaff J, et al. Preferred reporting items for systematic reviews and meta-analyses: the PRISMA statement. Ann Intern Med 2009;151:264-9.

32. Stang A. Critical evaluation of the Newcastle-Ottawa Scale for the assessment of the quality of nonrandomized studies in metaanalysis. Eur J Epidemiol 2010;25:603-5.

33. Li JJ, Li HY, Gu F. Diagnostic significance of serum osteopontin level for pancreatic cancer: a meta-analysis. Genet Test Mol Biomarkers 2014;18:580-6.

34. DerSimonian R, Kacker R. Random-effects model for meta-analysis of clinical trials: an update. Contemp Clin Trials 2007;28:105-14.

35. Duval S. The trim and fill method. In: Rothstein HR, Sutton AJ, Borenstein M. eds. Publication bias in meta-analysis-prevention, assessment and adjustments. Chichester: John Wiley \& Sons Ltd, 2005:127-44.

36. Ye C, Ruan Y, Zou L, et al. The 2011 survey on hypertensive disorders of pregnancy (HDP) in China: prevalence, risk factors, complications, pregnancy and perinatal outcomes. PLOS ONE 2014;9:e100180.

37. Ghulmiyyah L, Sibai B. Maternal mortality from preeclampsia/ eclampsia. Semin Perinatol 2012;36:56-9.

38. Ranta JK, Raatikainen K, Romppanen J, et al. Decreased PAPP-A is associated with preeclampsia, premature delivery and small for gestational age infants but not with placental abruption. Eur J Obstet Gynecol Reprod Biol 2011;157:48-52.

39. Yang $\mathrm{Y}, \mathrm{Su} \mathrm{X}, \mathrm{Xu} \mathrm{W}$, et al. Interleukin-18 and interferon gamma levels in preeclampsia: a systematic review and meta-Analysis. Am J Reprod Immunol 2014;72:504-14. 